\title{
N-Heterocyclic Carbenes as Activating Ligands for Hydrogenation and Isomerization of Unactivated Olefins
}

\author{
Ureshini L. Dharmasena, Heather M. Foucault, Eduardo N. dos Santos, ${ }^{\dagger}$ Deryn E. Fogg,* and \\ Steven P. Nolan \\ Center for Catalysis Research and Innovation, Department of Chemistry, University of Ottawa, \\ 10 Marie Curie, Ottawa, ON, Canada, K1N 6N5.
}

\section{SUPPORTING INFORMATION:}

Contents: Experimental and spectroscopic data for NHC complexes $\mathbf{4 a}$ and $\mathbf{4 b}$, general procedures for hydrogenation experiments, and composition profiles for reduction of cyclododecene $\left(140 \mathrm{psi}_{2}, 80^{\circ} \mathrm{C}\right)$ and allylbenzene $\left(50 \mathrm{psi}_{2}, 80^{\circ} \mathrm{C}\right)$.

\footnotetext{
* Corresponding author. E-mail: dfogg@science.uottawa.ca. Fax: (613) 562-5170

† Sabbatical visitor from Departamento de Química - ICEx, Universidade Federale de Minas Gerais, 31270-901 Belo Horizonte, Brazil.

Department of Chemistry, University of New Orleans, New Orleans, LA 70148.
} 
General Procedures. Reactions were carried out at RT $\left(22^{\circ} \mathrm{C}\right)$ under $\mathrm{N}_{2}$ using standard Schlenk or drybox techniques. Dry, oxygen-free solvents were obtained using an Anhydrous Engineering solvent purification system, and stored over Linde $4 \AA$ molecular sieves. $\mathrm{CDCl}_{3}$ and $\mathrm{C}_{6} \mathrm{D}_{6}$ were dried over activated sieves (Linde $4 \AA$ ) and degassed by consecutive freeze/pump/thaw cycles. $\mathrm{RuHCl}(\mathrm{CO})\left(\mathrm{PPh}_{3}\right)_{3} 3,{ }^{1} \mathrm{IMes},{ }^{2}$ and $\mathrm{H}_{2} \mathrm{IMes}^{2}$ were prepared according to the literature methods. Substrates were purified by passage through neutral alumina, followed by vacuum distillation. ${ }^{1} \mathrm{H}$ NMR (300 MHz), ${ }^{31} \mathrm{P}$ NMR $(121 \mathrm{MHz})$ and ${ }^{13} \mathrm{C}(75 \mathrm{MHz})$ spectra were recorded on a Bruker Avance-300 spectrometer. NMR spectra are reported relative to external $85 \% \mathrm{H}_{3} \mathrm{PO}_{4}\left({ }^{31} \mathrm{P}\right)$ or TMS $\left({ }^{1} \mathrm{H},{ }^{13} \mathrm{C}\right)$ at $0 \mathrm{ppm}$. IR spectra were measured on a Bomem MB100 IR spectrometer. Inertatmosphere MALDI-MS analyses were performed using a Bruker OmniFlex MALDI-TOF mass spectrometer equipped with a nitrogen laser $(337 \mathrm{~nm})$, and interfaced to an MBraun glovebox. Data were collected in positive reflectron mode, with the accelerating voltage held at $20 \mathrm{kV}$. Matrix (anthracene) and analyte solutions were prepared in $\mathrm{CH}_{2} \mathrm{Cl}_{2}$ at concentrations of 20 $\mathrm{mg} / \mathrm{mL}$ and $1 \mathrm{mg} / \mathrm{mL}$, respectively. Samples were mixed in a matrix:analyte ratio of 20:1. Microanalyses were carried out by Guelph Chemical Laboratories Ltd., Guelph, Ontario.

$\operatorname{RuHCl}(\mathbf{C O})(\mathrm{IMes})\left(\mathbf{P P h}_{3}\right)$ 4a. A solution of IMes $(188 \mathrm{mg}, 0.62 \mathrm{mmol})$ in $8 \mathrm{~mL}$ toluene was added to a suspension of $\mathrm{RuHCl}(\mathrm{CO})\left(\mathrm{PPh}_{3}\right)_{3}(420 \mathrm{mg}, 0.44 \mathrm{mmol})$ in $8 \mathrm{~mL}$ toluene. The solution was stirred at $22{ }^{\circ} \mathrm{C}$ for $3 \mathrm{~h}$, after which time reaction was complete, as indicated by ${ }^{31} \mathrm{P}$ NMR analysis. The solvent was concentrated under vacuum to ca. $0.5 \mathrm{~mL}$, treated with hexanes, and chilled to $-35^{\circ} \mathrm{C}$. A yellow-orange microcrystalline precipitate was obtained, which was filtered off, washed with cold hexanes $(3 \times 5 \mathrm{~mL})$ and dried under vacuum. Yield $0.275 \mathrm{~g}(85 \%)$. ${ }^{1} \mathrm{H}$ NMR $\left(\mathrm{C}_{6} \mathrm{D}_{6}, 298 \mathrm{~K}\right)$ : $\delta$ 7.53-7.49 and $7.02-6.94(\mathrm{~m}, 15 \mathrm{H}, \mathrm{Ar}), 6.78$ (s, 2H, IMes Ar, m-CH), 
6.73 (s, 2H, IMes Ar, m-CH), 6.24 (s, 2H, IMes $\mathrm{CH}$ ), 2.44 (s, 6H, $\mathrm{CH}_{3}$ ), $2.32\left(\mathrm{~s}, 6 \mathrm{H}, \mathrm{CH}_{3}\right), 2.10$ $\left(\mathrm{s}, 6 \mathrm{H}, \mathrm{CH}_{3}\right),-23.89\left(\mathrm{~d},{ }^{2} J_{\mathrm{PH}}=24.3 \mathrm{~Hz}, 1 \mathrm{H}, \mathrm{Ru} H\right) .{ }^{31} \mathrm{P}\left\{{ }^{1} \mathrm{H}\right\} \mathrm{NMR}\left(\mathrm{C}_{6} \mathrm{D}_{6}\right): \delta 42.1\left(\mathrm{~s}, \mathrm{PPh}_{3}\right)$. ${ }^{13} \mathrm{C}\left\{{ }^{1} \mathrm{H}\right\} \operatorname{NMR}\left(\mathrm{C}_{6} \mathrm{D}_{6}\right): \delta 201.4\left(\mathrm{~d}, C \mathrm{O},{ }^{2} J_{\mathrm{PC}}=14.4 \mathrm{~Hz}\right), 189.7\left(\mathrm{~d}, \mathrm{NCN},{ }^{2} J_{\mathrm{PC}}=103.6 \mathrm{~Hz}\right), 138.2$ to $127.5\left(\mathrm{Ph} C \mathrm{H}\right.$ ), 122.1 (s, $\mathrm{NCHCHN}$ ), 20.8 (s, Mes $\mathrm{CH}_{3}$ ), 18.7 (s, Mes $\mathrm{CH}_{3}$ ), 18.6 (s, Mes $\mathrm{CH}_{3}$ ). IR (Nujol): v(CO) 1913, v(Ru-H) $1897 \mathrm{~cm}^{-1}$. MALDI-MS, m/z: Calcd for [M-H] $]^{+}, 731.3$; Found, 731.1. Anal. Calcd. for $\mathrm{C}_{40} \mathrm{H}_{40} \mathrm{ClN}_{2} \mathrm{OPRu}$ : C, 65.61; H, 5.51; N, 3.82\%. Found: C, 65.13; H, 5.21; $\mathrm{N}, 3.39 \%$.

$\operatorname{RuHCl}(\mathbf{C O})\left(\mathrm{H}_{2} \mathrm{IMes}\right)\left(\mathbf{P P h}_{3}\right) 4 \mathrm{~b}$. A solution of $\mathrm{H}_{2} \mathrm{IMes}$ was generated in situ by addition of lithium tert-butoxide (101 $\mathrm{mg}, 1.26 \mathrm{mmol})$ to a solution of $\mathrm{H}_{2} \mathrm{IMes} \bullet \mathrm{HCl}(305 \mathrm{mg}, 1.26 \mathrm{mmol})$ in $25 \mathrm{~mL}$ THF. The solution was stirred at $22{ }^{\circ} \mathrm{C}$ for $2 \mathrm{~h}$, then added dropwise to a suspension of $\mathrm{RuHCl}(\mathrm{CO})\left(\mathrm{PPh}_{3}\right)_{3}(600 \mathrm{mg}, 0.629 \mathrm{mmol})$ in $30 \mathrm{~mL}$ THF. The reaction mixture was stirred for 3 h at $22{ }^{\circ} \mathrm{C}$, after which time reaction was complete, as judged by ${ }^{31} \mathrm{P}$ NMR analysis. The solution was filtered (Celite), concentrated, treated with hexanes, and chilled to $-35{ }^{\circ} \mathrm{C}$ to precipitate a bright yellow microcrystalline product. The solid was filtered off, washed with cold hexanes $(3 \times$ $5 \mathrm{~mL})$, and dried under vacuum. Yield $376 \mathrm{mg}(82 \%) .{ }^{1} \mathrm{H}$ NMR $\left(\mathrm{C}_{6} \mathrm{D}_{6}, 298 \mathrm{~K}\right): \delta 7.38-7.45$ and $6.88-6.93$ (m, 15H, Ar), 6.80 (s, 2H, $\mathrm{H}_{2} \mathrm{IMes} \mathrm{Ar}, \mathrm{m}-\mathrm{CH}$ ), 6.76 (s, 2H, $\left.\mathrm{H}_{2} \mathrm{IMes} \mathrm{Ar}, \mathrm{m}-\mathrm{CH}\right), 3.25$ (s, 4H, $\left.\mathrm{H}_{2} \mathrm{IMes} \mathrm{CH}_{2}\right), 2.63\left(\mathrm{~s}, 6 \mathrm{H}, \mathrm{CH}_{3}\right), 2.49\left(\mathrm{~s}, 6 \mathrm{H}, \mathrm{CH}_{3}\right), 2.10\left(\mathrm{~s}, 6 \mathrm{H}, \mathrm{CH}_{3}\right),-24.00\left(\mathrm{~d},{ }^{2} J_{\mathrm{PH}}=\right.$ $24.0 \mathrm{~Hz}, 1 \mathrm{H}, \mathrm{Ru} H) .{ }^{31} \mathrm{P}\left\{{ }^{1} \mathrm{H}\right\} \mathrm{NMR}\left(\mathrm{C}_{6} \mathrm{D}_{6}\right): \delta 41.0\left(\mathrm{~s}, \mathrm{PPh}_{3}\right) .{ }^{13} \mathrm{C}\left\{{ }^{1} \mathrm{H}\right\} \mathrm{NMR}\left(\mathrm{CDCl}_{3}\right): \delta 216.0(\mathrm{~d}$, $\left.\mathrm{N} C \mathrm{~N},{ }^{2} J_{\mathrm{PC}}=95.8 \mathrm{~Hz}\right), 200.8\left(\mathrm{~d}, C \mathrm{O},{ }^{2} J_{\mathrm{PC}}=14.0 \mathrm{~Hz}\right), 137.7$ to $127.6(\mathrm{Ph} C \mathrm{H}), 51.3(\mathrm{~s}$, $\mathrm{NCH}_{2} \mathrm{CH}_{2} \mathrm{~N}$ ), $51.2\left(\mathrm{~s}, \mathrm{NCH}_{2} \mathrm{CH}_{2} \mathrm{~N}\right.$ ), 21.2 (s, Mes $\mathrm{CH}_{3}$ ), 18.9 (s, Mes $\mathrm{CH}_{3}$ ). IR (Nujol): v(CO) 1911, v(Ru-H) $1865 \mathrm{~cm}^{-1}$. MALDI-MS, m/z: Calcd for [M] $]^{+}$, 734.3; Found, 734.1. Anal. Calcd. for $\mathrm{C}_{40} \mathrm{H}_{42} \mathrm{ClN}_{2} \mathrm{OPRu}: \mathrm{C}, 65.43 ; \mathrm{H}, 5.76 ; \mathrm{N}, 3.82 \%$. Found: $\mathrm{C}, 65.40 ; \mathrm{H}, 5.57 ; \mathrm{N}, 3.84 \%$. 
General Procedure for Catalytic Hydrogenation Experiments. A representative procedure is given for hydrogenation of cyclododecene. In a glovebox, a Parr autoclave was charged with cyclododecene $(0.333 \mathrm{~g}, 2.00 \mathrm{mmol})$, toluene $(15 \mathrm{~mL})$, and $\mathbf{4 b}(0.73 \mathrm{mg}, 1.00 \mu \mathrm{mol})$. The autoclave was removed from the drybox, purged with $\mathrm{H}_{2}$ (while stirring), pressurized to $140 \mathrm{psi}$, and heated to $80^{\circ} \mathrm{C}$. The sample reached thermal equilibrium in seven minutes, at which point the sample designated $\mathrm{t}=0 \mathrm{~h}$ was removed. Subsequent samples were taken at $30 \mathrm{~min}, 1 \mathrm{~h}$ and 2 h. Product yields were determined by gas chromatographic analysis and integrated against tetralin as an internal standard. An average of two trials is reported $( \pm 3 \%)$.

\section{References.}

(1) Ahmad, N.; Levison, J. J.; Robinson, S. D.; Uttley, M. F. Inorg. Synth. 1974, 15, 48.

(2) Arduengo, A. J.; Krafczyk, R.; Schmutzler, R.; Craig, H. A.; Goerlich, J. R.; Marshall, W. J.; Unverzagt, M. Tetrahedron 1999, 55, 14523. 


\section{Conversion profiles for Representative Reactions.}

Cyclododecene reduction $\left(140 \mathrm{psi}_{2}, 80{ }^{\circ} \mathrm{C},[\mathrm{S}]:[\mathrm{C}]=2000: 1,1.00 \mu \mathrm{mol} \mathrm{Ru}, 15 \mathrm{~mL}\right.$ toluene). Key: yellow, cis-cyclododecene; purple, trans-cyclododecene; blue, cyclododecane product.
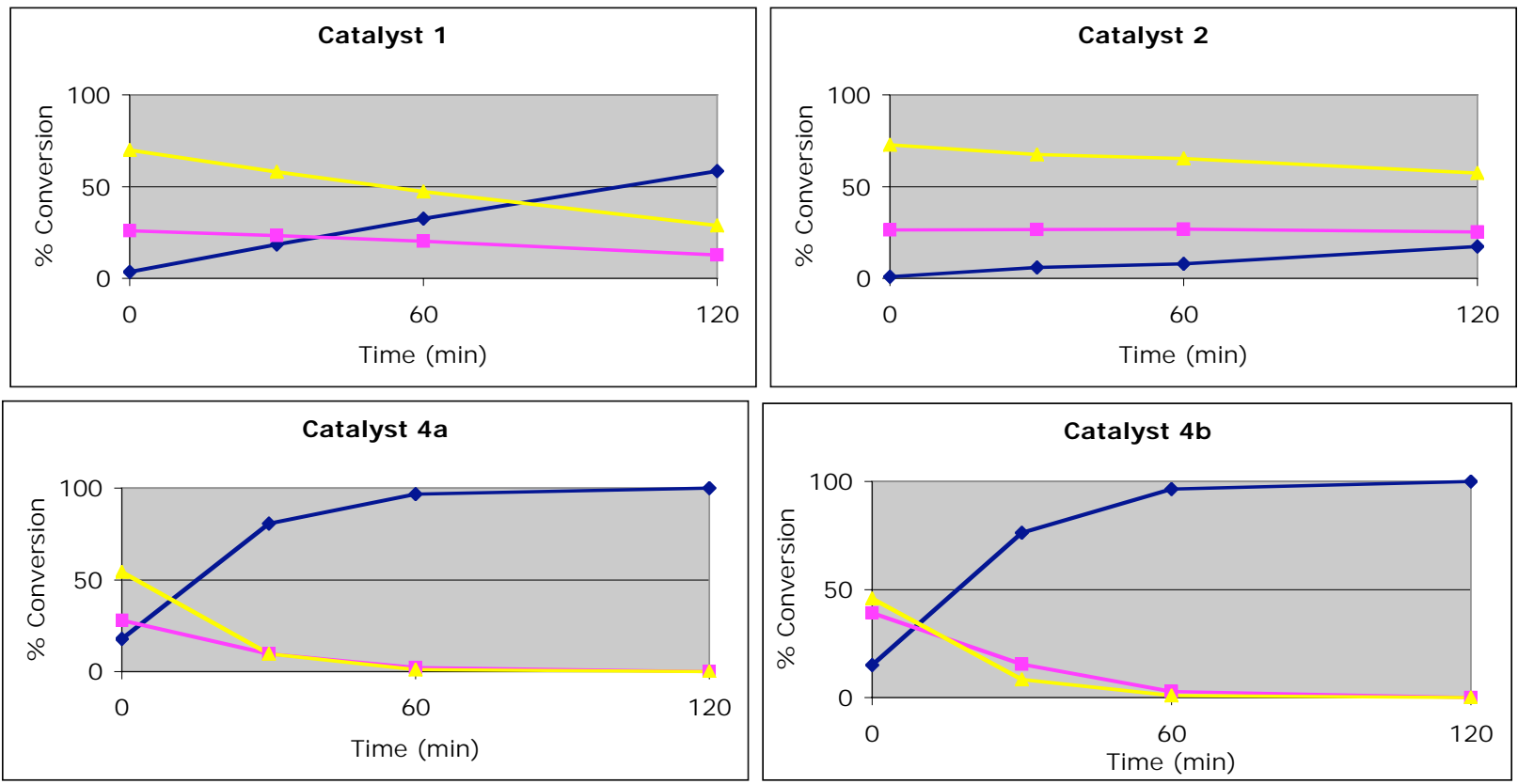

Allylbenzene reduction $\left(50 \mathrm{psi}_{2}, 80{ }^{\circ} \mathrm{C},[\mathrm{S}]:[\mathrm{C}]=2000: 1,1.00 \mu \mathrm{mol} \mathrm{Ru}, 15 \mathrm{~mL}\right.$ toluene). Key: red, allylbenzene; yellow, cis- $\mathrm{PhC}(\mathrm{H})=\mathrm{CHMe}$; purple, trans $-\mathrm{PhC}(\mathrm{H})=\mathrm{CHMe}$; blue, propylbenzene.
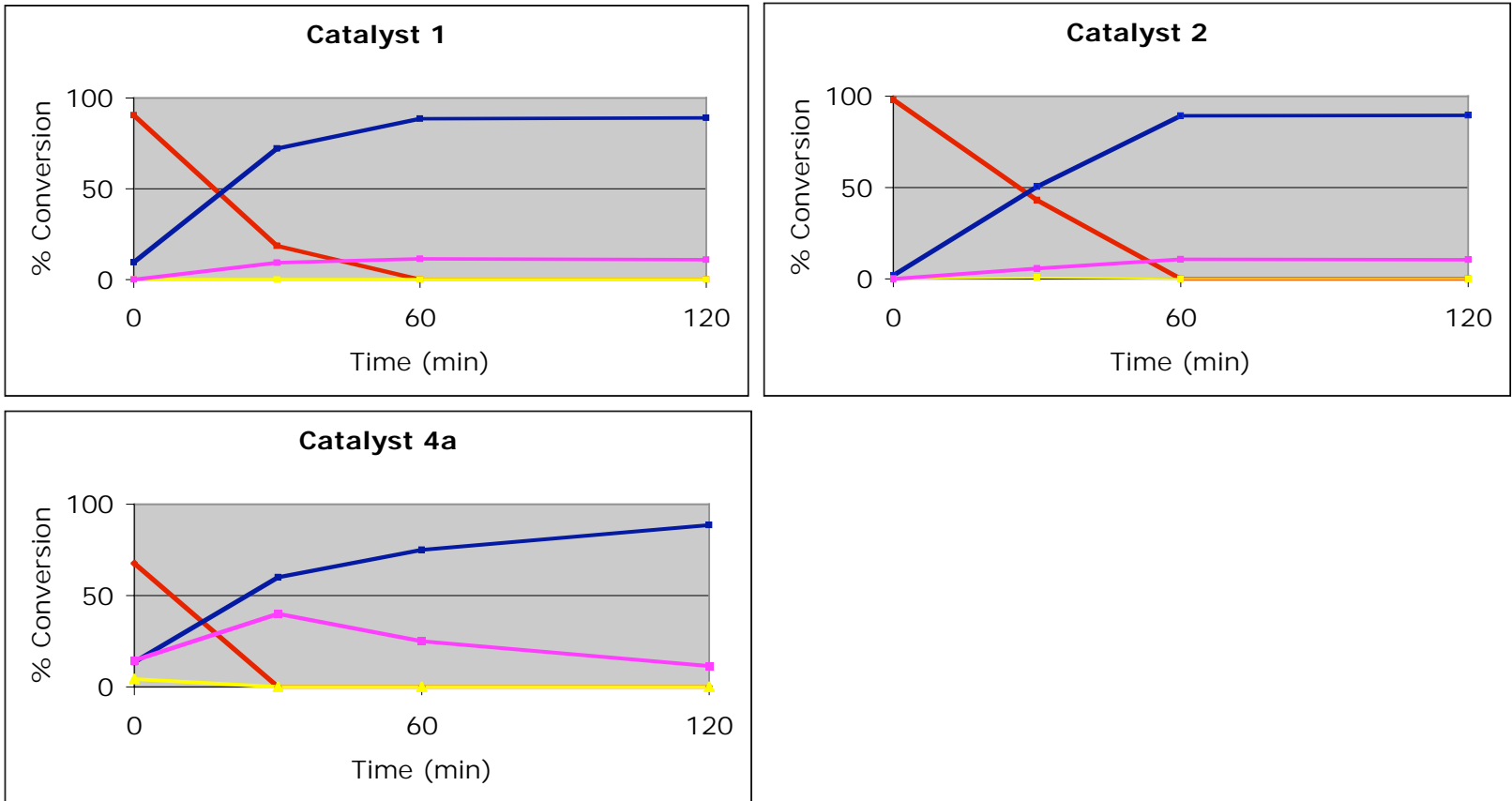